\title{
A Photoelectric Followup and Recording System, and its Application to Remote Observations of the Beam in High-Precision Balances
}

\author{
H. A. Bowman and L. B. Macurdy
}

(July 22, 1959)

\begin{abstract}
A device is described which permits a significant increase in the frequency of the oscillations of the beam of a balance, thereby shortening the interval during which upsetting ambient conditions can act. The device is a servo-driven photoelectric followup system responding to the position-modulated light signal reflected from a mirror attached to the balance $12 \mathrm{ft}$ away from the photocell. Reproducibility in observing the position of the light beam is $0.001 \mathrm{in}$. or less.
\end{abstract}

\section{Introduction}

For the past 10 years the NBS Mass Unit has been studying the causes of uncertainties in highprecision mass determinations. These studies have demonstrated that causes of major uncertainties are due to variations in ambient conditions occurring during the experimental interval. Exclusive of the time required by the preparations for a mass-determination experiment, such as cleaning weights, and exclusive of computation time, about one-third of the experimental interval is required for manipulating the weights and the balance, and about two-thirds for the actual swinging of the beam. The virtues of a radically decreased beam period are that the interval during which ambient changes can act is reduced; the adjustments required to control the period of oscillation of the beam are simple. The reason why short periods are conventionally rejected is that the square of the period, to a good approximation, is proportional to the balance sensitivity (deflection/change of mass). This must not be confused with the weighing precision that can be achieved. For assessing the latter, a short period presents an important advantage in that the on-scale range of the balance is increased, thereby permitting the use of larger (and hence more accurate) sensitivity weights.

The most serious disadvantage of a fast-swinging beam is the difficulty of maintaining the accuracy of the turning-point observations. If the overall experimental precision is to be maintained, turning point data must be more accurate with a fastswinging beam than with a slow one due to the fact that the balance sensitivity is less for a fast-swinging beam. These comments on beam period and observational accuracy are general and apply to all equal arm balances.

The desirability of an improved method of observing the motion of the balance beam has long been recognized. Photoelectric methods ${ }^{1}$ have previously been used; however, a survey of existing instrumentation of this type did not reveal devices capable of operating directly in mass-determination work where precision of the very highest order was required.

Design requirements for an instrument considered suitable for NBS Mass Laboratory studies are as follows:

a. The device should be capable of defining the position of a light beam to a few ten thousandths of an inch at the end of a 12 -ft optical lever.

b. It should have freedom from zero drift over long periods (of several days) from either thermal or electrical causes; or, if such drift exists, it should be capable of being measured by independent means.

c. The data must be available in numerical form for computation or in a form suitable as an input for a strip chart recorder.

d. There should be a surplus of mechanical power, directly related to beam motion, for the operation of automatic computation or data-handling devices or for manipulation of various balance-control mechanisms.

e. The device should be capable of operating in normal fluorescent illumination.

f. The design should use, insofar as practicable, commercially available assemblies or subassemblies.

g. The design configuration should be sufficiently flexible to permit its use in several different applications required in mass-laboratory studies.

A photoelectric device was fabricated which acceptably meets the above requirements. Completed about a year ago, it has been a principal tool in a series of design studies preliminary to the construction of a high-speed automatic weighing system of

$1 \mathrm{~V}$. E. Birgel, Installation for the experimental investigation of precision balances, Techniques of Measurement, No. 1, p. 43 (Jan. 1956); C. Norman Cochran, Automatic recording vacuum microbalance, Rev. Sci. Instr. 29, Cochran, Automatic
No. 12 (Dec. 1958) 
the highest attainable precision. At the completion of these studies the device, with modifications, will become a permanent part of this proposed weighing system.

\section{Description of the Instrument}

This automatic observing device may be assembled in several different forms, the essential features of one of which are shown in figure 1. The photoelectric light source is a single tungsten-filament bulb excited by a well regulated and filtered d-c power supply. The light from this bulb is reflected from an off-axis mounted mirror on the shaft of a 3,600rpm synchronous motor driven from the 60-cycle commercial power line, through a high-quality achromatic condenser lens to a mirror mounted on the beam of the balance under observation. The balancebeam mirror reflects this light to a photoelectric cell carried to and fro on a lead screw driven by a twophase motor through a rubber-tire and friction-disk assembly. The image of the tungsten filament, brought to focus by the condenser in the photocathode plane, is oriented vertically by appropriate positioning of the bulb. The 3,600-rpm synchronous motor imparts a small amount of 60-cps nutation to the light beam which manifests itself as an apparent small side-to-side motion of the light beam across the face of the photocathode parallel to the length of the lead screw. When the photocell lies in the center of this nutation it is illuminated 120 times per second, and the resulting 120-cps output is dissipated in a 60-cps filter immediately following. If, however, the photocell is off-center in the nutation its output con-

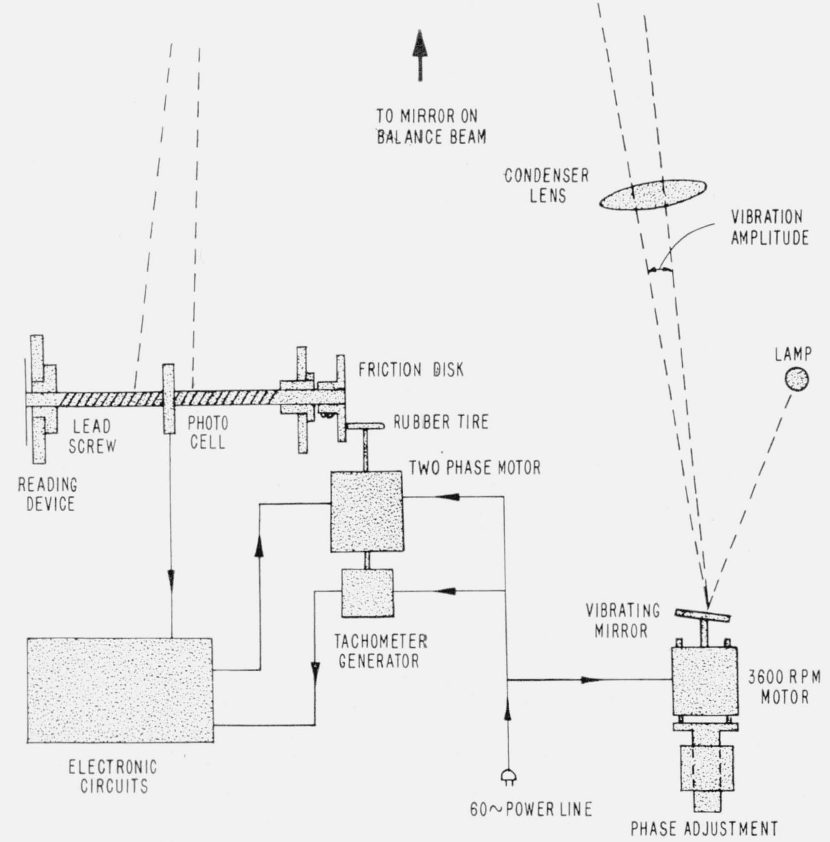

Figure 1. Schematic diagram of the essential elements of the balance observing device (when assembled to provide turning point data). tains a 60 -cycle component, the phase and magnitude of which are functions of the direction and amount of this offcenter displacement. This component is amplified and applied to the control winding of the two-phase motor. When the phase of the nutation is properly adjusted with respect to the 60-cycle power line, the motor rotates the lead screw in the direction necessary to re-center the photocell in the light beam. When the balance beam swings, the nutating light beam moves back and forth along the length of the lead screw, and the motion of the balance beam is determined from observations on the angular position of the lead screw.

\subsection{Optical System}

In any measuring system in which the smallest readable unit is evaluated in terms of the quantity measured by observations widely separated on the scale, great care must be taken to assure that the various optical elements in the system do not distort the relative lengths of the scale units. In weighing, sensitivity weights are added to the major loads on the pans to permit balance indications to be converted into mass units. Distortion arises from parallax in eyepieces and from the various windows and mirrors which are not perfectly flat. As the magnification of the observing system increases, distortion increases. In the NBS system all mirrors are made by evaporating aluminum films on optical flats. Windows are polished flat and the two surfaces are maintained parallel. They are coated with magnesium fluoride to reduce reflection losses. There is only one lens in the system, which is an $\mathrm{f} / 5$ achromatic objective of $25 \mathrm{in}$. in focal length.

Requirements for maximum-displacement sensitivity are equality in the image plane of the width of the image of the tungsten filament, of the width of the photocathode, and of the peak amplitude of the nutation. The width of the photocathode is slightly less than 1116 in. The condenser lens magnifies the image of the 0.005 -in. diameter filament to about $1 / 16$ in. at the extremity of the 310 -in. image distance. The amplitude of the nutation is adjustable by varying the amount of off-axis displacement of the mirror on the 3,600-rpm synchronous motor, as shown on figure 2 .

The photosensitive unit is a cadmium selenide photoconductive cell, the peak response of which lies in the red region. This photocell used with a tungsten-filament light source makes an excellent combination for use in a room which is illuminated with fluorescent lighting (largely blue response). The collimator in front of the cell is merely a $1 / 2-i n$. diameter tube 3 -in. long with a blackened inner wall. Some experiments indicate that the predominately red light from the tungsten filament may be thermally upsetting to the balance. Additional disadvantages of the red-sensitive system currently in use are the low frequency response of the cadmium selenide cell, ${ }^{2}$ and the sensitivity of this cell to

2 The time constant at $0.01-\mathrm{fc}$ illumination is $0.3 \mathrm{sec}$, at $0.1 \mathrm{fc}$ it is $0.07 \mathrm{sec}$, and at 1.0 it is 0.017 sec. From trade data published by the Clairex Corp., New York, N. Y. 


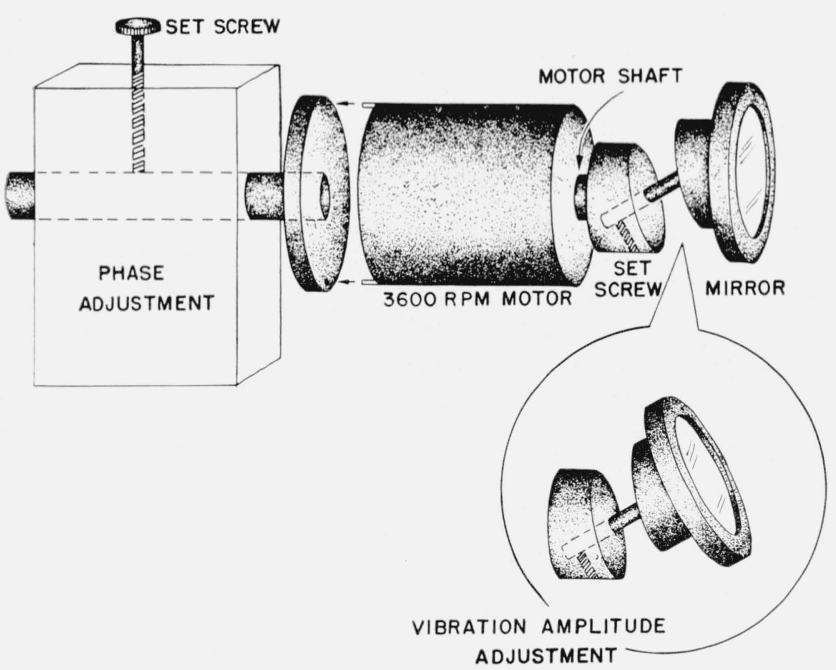

FIGURE 2. The synchronous motor-supporting assembly which provides both amplitude and phase control of the nutation of the light beam.

temperature changes. Experiments with blue sensitive systems (high-frequency vacuum photodiodes) were successful and the present system could be so modified.

Light beam nutation is presently achieved by the rotating mirror shown in figure 2 . For applications requiring that the light source be rigidly attached to the balance case an alternative method of nutating the light beam must be used, since the vibration associated with the 3,600-rpm motor is greater than could be tolerated in a high precision balance. There are two satisfactory methods of obtaining a carrier signal, both of which are vibration free. One method requires (a-c) excitation of the tungsten filament in a suitably-oriented magnetic field. Under these conditions the tungsten filament vibrates back and forth at an amplitude controlled by the magnitude of the field. A variation of this method is d-c filament excitation in the presence of a-c magnetic field. The effect of the vibration of the lamp filament is vanishingly small when related to balance operation; however, a different method of attaining a carrier was successfully tried over short optical paths. A neon pilot lamp, excited by the power line, provided illumination in the pass band of the photocell. The image of the two electrodes was brought to focus in the plane of the photocathode, and the action of the servosystem immediately centered the photocell in the dark space between the two electrode images.

\subsection{Mechanical System}

The heart of the mechanical system is the lead screw, figure 1, which replaces the engraved scales used in high-quality telescope-observing systems. Its threaded length is $20 \mathrm{in}$. with a $1 \frac{1}{2}$ in. shank at each end. The major diameter of the thread is $1 / 2$ in. and of the shanks $1 / 4$ in. It was ground from SAE No. 6150 chrome vanadium steel. Threads were lapped and polished until the lead error was well distributed along the entire threaded length. This lead error distribution was measured by the NBS Engineering Metrology Section to be a total of $0.0004 \mathrm{in}$. in $20 \mathrm{in.}$ It has a " 2 start, 10 pitch thread" (20 lands and 20 grooves per inch) with a $60^{\circ}$ profile.

The driven member is a bronze half nut with straight milled ridges meshing with the top surface of the lead screw. These ridges are relieved at both the crests and troughs, thereby assuring that driving contact is maintained with the polished $60^{\circ}$ thread surfaces. This bronze member is attached (at the appropriate angle) to the carriage of the photocell. The photocell carriage is supported at three pointstwo ball bushings around a rod mounted parallel to the lead screw and the bronze driven-member riding on the lead screw itself.

The lead-screw shanks ride in bronze bushings mounted in heavy-brass end plates which are, in turn, rigidly attached to a lathe bed obtained for the purpose. At one end of the lead screw a friction disk is mounted whose bore has been reamed to match the diameter of the shank. The friction surface (on which the rubber-tire drive wheel bears) was turned normal to this reamed bore, and faces away from the threaded portion of the lead screw. The portion of the back surface of this disk which bears against the collar of the supporting-bronze bushing (see fig. 1) is also turned normal to the bore. The drive wheel then holds the friction disk in constant contact with this thrust collar and the reverse surface of the friction disk defines the reference surface from which all displacement measurements on the photocell are made. The shank at the opposite end of the lead screw is free to move in its bushing either longitudinally (under thermal forces on the lead screw) or rotationally.

The shaft of the two-phase motor is extended and is fitted with a rubber-tire wheel which can drive the friction disk at any desired radius. This radius is selected to provide gear reduction between the twophase motor and the photocell carriage sufficient to insure that the motor will operate near the upper limit of its speed, and will, of course, depend upon the amplitude and peak velocity of the gross motion of the photocell carriage. A bronze radial bushing can be slid to any position on the extended shaft of the two-phase motor to take up the radial load necessarily applied to the shaft by the friction drive. This bushing is not shown in figure 1, however, its necessity is obvious.

\subsection{Observing-Recording System}

One of the system design requirements, stated in the introduction, was that data be furnished in numerical form as well as in a form suitable for a strip-chart recorder. Numerical data on the angular position of the lead screw at the instant of the balance turning point are observed on a dial directly coupled to the lead screw. However, the motion of the lead screw is sufficiently rapid (even when going through a turning point), that an operator would experience 
great difficulty in estimating the extreme position of the dial attached to the end of the lead screw in the process.

In order to provide the operator sufficient time to observe and make an accurate estimate of this extreme angular position, there is one turn or $0.1 \mathrm{in}$. of free play between the lead screw and this dial. The dial is therefore motionless during the interval that the lead screw makes a complete revolution after the turning point is reached. This dial is engraved with 100 index marks spaced about 1/8 in. apart, each interval representing $0.001 \mathrm{in}$. of linear motion of the photocell carriage. When this dial is stopped with turning-point data showing, the operator is able to make an unhurried estimate to one or two ten-thousandths of an inch of the true position of the turning point. Turning-point data in inches and tenths of an inch are observed on a second dial geared down by a ratio of 20 from the lead screw. Thus, data to six significant figures are obtained, three figures on each of two dials.

There are on the market several data-taking disk systems designed to be driven by lead screws or other rotating members. Such systems automatically type turning-point data or record corresponding binary or decade information on magnetic tape or IBM punch cards. The drive mechanism for the lead screw was designed with the possibility in mind of later installing one of these automatic data-taking devices, hence there is available sufficient torque to drive any of the numerous types currently available.

When it is desired to obtain a continuous record of balance-beam motion on a strip chart recorder, a high-linearity multiple-turn servo-type potentiometer is coupled to the lead screw. A d-c voltage is then impressed across this potentiometer and with two wire-wound resistors a Wheatstone bridge circuit is formed, with the recorder occupying the position of the galvanometer.

\subsection{Electronic System}

The electronic system is divided into two groups. The first group contains the d-c power supplies and the power amplifying circuitry. There are many vacuum tubes associated with these functions, as well as power resistors and transformers, all of which generate large quantities of heat. Accordingly, the first group of electronics is located remotely from the lead screw and balances and is coupled to the second group by a shielded cable.

The second group of electronic circuits is mounted in a small chassis next to the lead screw. There are only two small electrometer tubes in this chassis which together dissipate only about $3 \mathrm{w}$. One tube is an amplifier with the high impedance cadmium selenide cell in the grid circuit, and the other is a cathode follower which provides a low impedance signal to the long cable connected with the power amplifying circuits located remotely from the lead screw. The chassis at the lead screw also contains the necessary switches, adjustable resistors, etc., to phase properly the various error signals, feedback voltages, etc.
Most of the major electronic components are high quality commercially available units. Every effort was made to keep signal lines shielded to guard against hum pickup, and manufacturer's recommendations as to grounding were carefully followed.

\subsection{Thermal Considerations}

Undesired thermal effects exist in almost all highprecision measuring systems. By careful placement of system components they are largely under control in the NBS balance observing device. In this apparatus, the main source of heat is the electronic power circuits, and as explained above, this group is located away from the lead screw. The two phase motor dissipates rather large quantities of heat, and, unfortunately, it must be located in fairly close proximity to the screw. In order to protect the screw, the motor is surrounded by a copper tube cooling jacket, through which water is circulated.

It was previously stated that photocell displacement measurements along the lead screw are made with respect to the thrust plane of the bronze thrust bearing at the drive end of the lead screw. The opposite end of the lead screw is free to slide in its radial bearing under thermal forces. In the same manner, the lathe bed upon which the lead screw is mounted is bolted to the marble pier only at the thrust bearing end, the opposite end being free to move under whatever forces may operate. A fused silica (quartz) rod was mounted alongside the lead screw to monitor any thermal expansion. In use, it is rigidly attached to the end plate containing the lead-screw thrust bearing with a micrometer device for measuring changes in length of the lead screw with respect to its own nearly invariant length. In the original installation of this system, difficulties were traced by means of this rod to heat from the power electronics and the servomotor.

On a number of occasions the balance under observation has been left undisturbed and the position of the light beam recorded over intervals of two or three days. Under such circumstances the position of the beam is seen to move to and fro at an amplitude of several thousandths of an inch, as illustrated in figure 3 . The period of this motion is roughly diurnal due to motion of the building (probably caused by solar heating.)

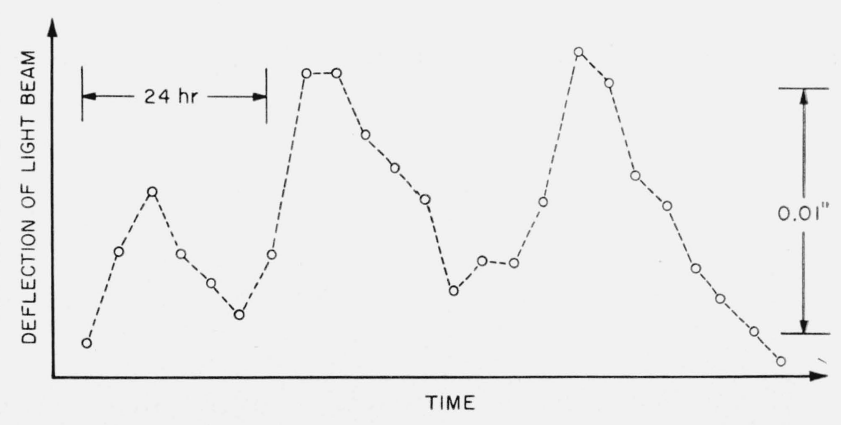

FIGURE 3. The motion of the light beam reflected from the mirror on the balance over a three-day interval. 
The only remaining troublesome heat source is the assembly (shown in fig. 2) comprising the 3,600-rpm synchronous motor and the tungsten-filament light source. This motor is kept running $24 \mathrm{hr}$ per day; however, the light source is turned down at night to a level sufficient only to maintain the photocell in the light beam under the loop action. When the lamp voltage is raised in the morning the recorder indicates a drift of about $0.003 \mathrm{in}$. in less than $1 \mathrm{hr}$. Thereafter there is no observable drift from this source. This drift may be a nonsymmetrical heating effect in the balance itself caused by the increased light level. Observations of this type resulted in the decision to use a heat filter and a multiplier phototube when this device is permanently fitted into an automatic high-speed weighing system. Consideration has been given to the use of a fused-silica lead screw; however, the reproducibility of existing balances does not now warrant this refinement.

\section{Use of the Instrument}

This device has been in use for about 1 yr in its present form during a study of the causes of uncertainties in balance operation. Data from these studies is not appropriate here; a few examples of the data presentation made by this device will be shown only to illustrate its operation. Figure 4a illustrates


Figure 4. Comparison of photoelectric and telescopic observation of the balance beam motion. the improvement in readability achieved when the photoelectric device is substituted for the conventional telescope system. The dotted curve is a plot of the left-hand turning points as recorded by an experienced observer through a high quality telescope system of 3 -in. aperture. The solid line is a plot of the same turning points but based upon photoelectrically taken data. Only the left-hand turning points are illustrated. When these left-hand turning points were combined with the associated righthand turning points (not shown), the computed balance indications were plotted and shown in figure $4 \mathrm{~b}$. The gentle downward drift of the balance indications computed from automatic data is ascribed to slowly changing thermal conditions in the balance case. During this experiment the period of the balance beam was about $20 \mathrm{sec}$.

Figure 5 shows two examples of balance indications computed from data by means of the device taken during experiments on various types of mechanical assemblies associated with high-precision balances.

Figure 6 shows a plot of balance-beam motion made on the strip-chart recorder when air turbulence

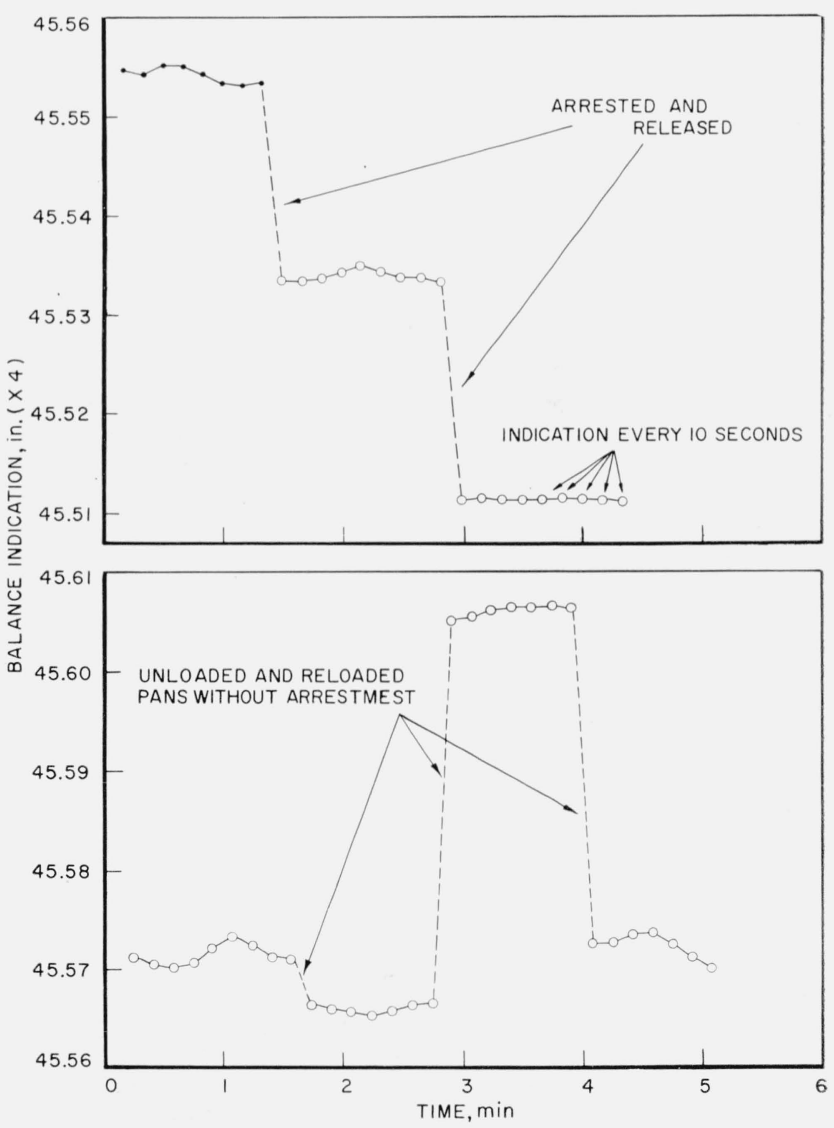

FIGURE 5. Illustrating mechanical errors in balance response: (above) arrestment error, (below) loading error.

Balance indication, plotted vertically, is numerically equal to 4 times the rest point. 


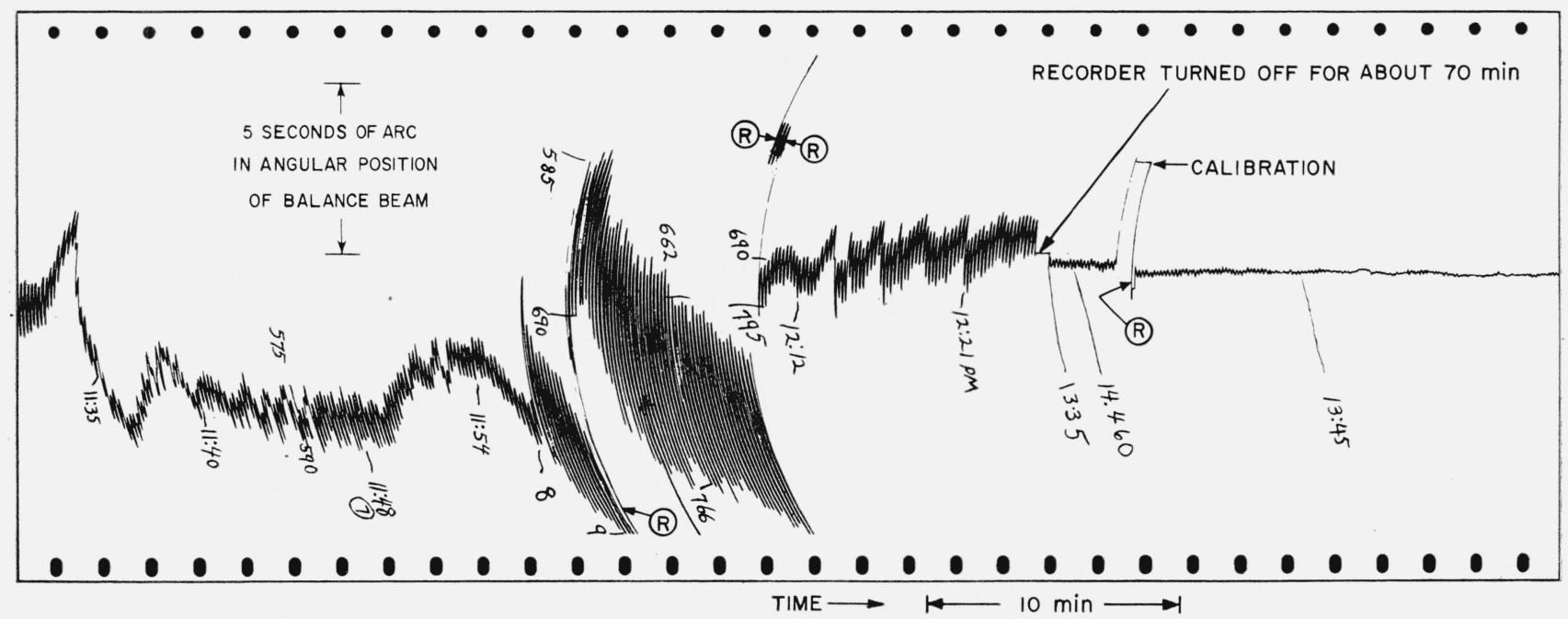

Figure 6. Balance response due to turbulent air in balance case.

The encircled $\mathrm{R}$ indicates that the pen was manually recentered.

existed in the balance case because of unfavorable thermal conditions. In the latter portion of the chart, the turbulence had subsided.

Consultation on electronic and servo problems was provided by M. L. Greenough of the Electronic Instrumentation Section. T. R. Young of the Optical
Instrument Section consulted on optical problems. The high precision mechanical work was performed by C. H. Hochgesang in the NBS Instrument Shop, under the direction of N. C. Pines.

$$
\text { Washington, D.C. }
$$

(Paper 63C2-11) 